\title{
Resistance of Bifidobacterium Isolated from Feces with or without Administration of Antibiotic-Resistant Bifidobacterium Preparation
}

\author{
Tsunesuke TOMODA $^{1}$ and Takashi KAgEYAMA ${ }^{2}$ \\ 'Department of Clinical Pathology and ${ }^{2}$ Second Department of Internal Medicine, Osaka Medical College, \\ Takatsuki, Osaka, 569 Japan
}

(Received for publication, September 29, 1995)

\begin{abstract}
The resistance of bifidobacteria to six kinds of antibiotics was examined. Bifidobacteria were isolated from the feces of healthy individuals (12 cases) and patients (11 cases) administered an antibiotic-resistant Bifidobacterium preparation (LacB$R^{\circledR}$ ). These patients were given antibiotic drugs etc. Only a few of the healthy individuals had the bifidobacteria resistant to ampicillin, cefazolin, erythromycin, minocycline and ofloxacin. However, many of the LacB- $\mathrm{R}^{\circledR}$-administered individuals had the bifidobacteria resistant to the antibiotics except minocycline and ofloxacin. All persons in both group showed resistance to amikacin. The administration of LacB$\mathbf{R}^{\circledR}$ was useful to maintain the number of intestinal Bifidobacterium because administered Bifidobacterium was recovered with the healthy control level in some of the patients receiving therapy with many kinds of antibiotics.
\end{abstract}

Key words: Bifidobacterium; antibiotic-resistant bifidobacteria; feces

Bifidobacterium has many useful actions as a component of the normal intestinal flora. Bifidobacterium preparations have also been given to patients during antibiotic chemotherapy to compensate for the disturbance of normal flora in intestine because bifidobacteria have primary resistance against a few antibiotics. We also reported some investigations for the usefulness of Bifidobacterium (2, 9). Recently, a Bifidobacterium preparation artificially given resistance against many antibiotics has been developed and applied clinically. However, clinical observations are required to determine the resistance to antibiotics in the Bifidobacterium.

In this study, the antibiotic resistance of Bifidobacterium isolated from feces was examined comparatively in two groups with or without the administration of a preparation of antibioticresistant Bifidobacterium (LacB-R ${ }^{\circledR}$ ) (Nikken Chemicals Co., Ltd.) (6). LacB- $\mathrm{R}^{\circledR}$ is a Bifidobacterium
(B. longum) preparation artificially given resistance to penicillins, cephalosporins, macrolides and aminoglycosides.

Twelve normal healthy adult persons and 11 patients with hematological malignancy who were being given antibiotic drugs, anticancer drugs, and immunosuppressive drugs were examined. LacB$R^{\circledR}$ was given $3 \mathrm{~g} /$ day for 4-6 weeks to the patient group.

The diluted $\left(10^{8} \times\right.$ dilution $)$ fecal samples were spread onto Mitsuoka's BS medium (5) and incubated anaerobically at $37^{\circ} \mathrm{C}$ for 3 days. After incubation, the colonies of Bifidobacterium species on BS medium were identified according to Bergey's classification (7). Some of the colonies of the same bifidobacteria in $10^{8} \times$ dilution sample were examined for resistance to 6 kinds of antibiotics. Then, the resistance of bifidobacteria examined in this report shows the data for dominant species $\left(>10^{8} / \mathrm{g}\right.$ feces) of bifidobacteria. 
Table 1. Antibiotic resistance and sensitivity of Bifidobacterium isolated from feces of LacB- $\mathrm{R}^{\mathbb{E}}$ administrated patients $(\mathrm{A}-\mathrm{K})$ and healthy individuals $(\mathrm{a}-1)$

\begin{tabular}{ccccccc}
\hline & Amp & Cef & Erythro & Amik & Mino & Oflo \\
\hline LacB-R $^{\circledR}$ & R & R & R & R & S & S \\
\hline A & R & R & R & R & S & S \\
B & R & R & R & R & S & S \\
C & R & R & R & R & S & S \\
D & R & R & R & R & R & R \\
E & R & R & R & R & R & S \\
F & R & R & R & R & S & R \\
G & R & R & R & R & S & R \\
H & R & R & S & R & S & S \\
I & R & S & R & R & S & S \\
J & S & S & S & R & S & R \\
K & S & S & R & R & S & S \\
\hline a & R & R & S & R & R & S \\
b & R & S & R & R & S & R \\
c & R & S & R & R & S & S \\
d & R & S & S & R & S & S \\
e & S & R & S & R & S & S \\
f & S & S & R & R & S & S \\
g & S & S & R & R & S & S \\
h & S & S & S & R & S & R \\
i & S & S & S & R & S & R \\
j & S & S & S & R & S & S \\
k & S & S & S & R & S & S \\
l & S & S & S & R & S & S \\
\hline
\end{tabular}

$\mathrm{R}$ : resistant, S: sensitive.

Drug resistance was tested by the disk diffusion method (Showa Disc ${ }^{\circledR}$ ). The kinds of antibiotics selected were ampicillin as penicillins (Amp), cefazolin as cephalosporins (Cef), erythromycin as macrolides (Erythro), amikacin as aminoglycosides (Amk), minocycline as tetracyclines (Mino) and ofloxacin as quinolones (Oflo). Resistance and sensitivity to antibiotics were determined from the inhibitory diameter formed around the disk. Bacteria were judged as resistant when the inhibitory zone of bifidobacterial growth in the circumference around the disk was not observed, as sensitive when an inhibitory zone was observed. The examination before LacB- $\mathrm{R}^{\circledR}$ administration could not be done because of receiving therapy with many antibiotics and many digestants already.

Table 1 shows the resistance against each antibiotic of Bifidobacterium in patients $(\mathrm{A}-\mathrm{K})$ and healthy individuals $(a-1)$. The bifidobacteria isolated from many persons in the LacB- $\mathrm{R}^{\circledR}$ administration group showed resistance to the antibiotics but not to minocycline. Three patients $(\mathrm{A}, \mathrm{B}, \mathrm{C})$ had the resistant bifidobacteria the same as LacB- $\mathrm{R}^{\circledR}$. Patient $\mathrm{D}$ showed resistance to all of the antibiotics tested. There were other cases of resistance to 5-2 kinds of antibiotics. However, the bifidobacteria isolated from many healthy individuals were not resistant to many kinds of antibiotics. Resistance to amikacin was seen in all cases in both groups.

The antibiotic resistance of Bifidobacterium has been reported by several investigators $(1,3,8,10)$, but not in detail because it is a nonpathogenic bacteria. The bifidobacteria have primary resistance to some drugs such as aminoglycosides and polymixin B, but are sensitive to penicillins, cephalosporins, macrolides, tetracyclines, etc. In this study, the bifidobacteria isolated from healthy individuals were resistant to a few antibiotics. However, the bifidobacteria isolated from LacB$\mathrm{R}^{\circledR}$-administered patients were resistant to many antibiotics, and there were 3 cases of resistance the same as LacB-R ${ }^{\circledast}$. Some bifidobacteria, however, did not have the same resistance as LacB$R^{\circledR}$. It may be derived from the acquisition of secondary resistance by the administration of many kinds of antibiotics or the existence of original Bifidobacterium.

Recently, quantitative or qualitative imbalance of normal intestinal flora by antibiotic therapy, or immunosuppressive drug therapy has been suggested, and Bifidobacterium administration has been carried out for the purpose of prophylaxis of these troubles. However, the bifidobacteria in the intestines of healthy persons are not always resistant against all antibiotics. If many antibiotics are given to persons having only original Bifidobacterium without antibiotic resistance, the growth of the Bifidobacterium in the intestines would be inhibited, and may have an unfavorable effect. Therefore, when many kinds of antibiotics are administered to patients with infectious diseases or in an immunosuppressive state, not only pathogenic bacteria but also normal flora are also diminished. Then, 
the simultaneous administration of the Bifidobacterium preparation $\left(\mathrm{LacB}-\mathrm{R}^{\circledR}\right)$ with resistance to many antibiotics would be useful to maintain the number of intestinal Bifidobacterium because the administered Bifidobacterium recovered to the healthy control level in some patients.

Miller et al (4) reported that the same species of Bifidobacterium also show differences in grade (minimal inhibitory concentration) of sensitivity to the same antibiotics. So testing the resistance to many antibiotics in strain of each Bifidobacterium isolated from the feces of the patients may also be required in administration of Bifidobacterium preparations and antibiotic drugs.

\section{REFERENCES}

(1) Aldrige KE, Sanders GV, Lewis AC and Marier RL: Susceptibility of anaerobic bacteria to beta-lactam antibiotics and beta-lactamase production. J Med Microbiol 16: 75-82, 1983

(2) Kageyama T, Tomoda T and Nakano Y: The effect of Bifidobacterium administration in patients with Leukemia. Bifidobacteria Microflora 3: 29-33, 1984

(3) Matteuzzi D, Crociani F and Brigidi P: Antimicrobial susceptibility of Bifidobacterium. Ann Microbiol Inst Past 134A: 339-349, 1983

(4) Miller LG and Finegold SM: Antibacterial sensitivity of Bifidobacterium (Lactobacillus bifidus). J Bacteriol 93: 125-130, 1967

(5) Mitsuoka T: A color atlas of anaerobic bacteria, Sobunsha, Tokyo, 1980 (in Japanese)

(6) Miyazaki K, Chida S, Akikawa K, Okamura N and Nakaya R: Isolation and characterization of the antibiotic-resistant strains of Bifidobacterium spp. Bifidobacteria Microflora 10: 33-41, 1991

(7) Sneath PHA, Mair NS, Sharpe ME and Holt JG: Bergey's manual of systematic bacteriology. Vol. 2. Williams \& Wilkins, Baltimore, 1986

(8) Shafran SD, Wong J and Chow AW: In vitro activity of Sch 34343 and cefbuperazone against anaerobic bacteria. Antimicrob Agents Chemother 27: 749752, 1985

(9) Tomoda T, Nakano Y and Kageyama T: Intestinal Candida overgrowth and Candida infection in patients with Leukemia (Effect of Bifidobacterium administration). Bifidobacteria Microflora 7: 71-74, 1988

(10) Whiting JL, Cheng $\mathrm{N}$ and Chow AW: Interactions of ciprofloxacin with clindamycin, metronidazole, cefoxitin, cefotaxime, and mezlocillin against grampositive and gram-negative anaerobic bacteria. Antimicrob Agents Chemother 31: 1379-1382, 1987 